\title{
Malan syndrome: Sotos-like overgrowth with de novo NFIX sequence variants and deletions in six new patients and a review of the literature
}

\author{
Merel Klaassens ${ }^{1,2,3}$, Deborah Morrogh ${ }^{3}$, Elisabeth M Rosser ${ }^{3}$, Fatima Jaffer ${ }^{4}$, Maaike Vreeburg ${ }^{2}$, \\ Levinus A Bok ${ }^{5}$, Tim Segboer ${ }^{6}$, Martine van Belzen ${ }^{6}$, Ros M Quinlivan ${ }^{4}$, Ajith Kumar ${ }^{3}$, Jane A Hurst ${ }^{3}$ \\ and Richard H Scottt,3,7
}

De novo monoallelic variants in NFIX cause two distinct syndromes. Whole gene deletions, nonsense variants and missense variants affecting the DNA-binding domain have been seen in association with a Sotos-like phenotype that we propose is referred to as Malan syndrome. Frameshift and splice-site variants thought to avoid nonsense-mediated RNA decay have been seen in Marshall-Smith syndrome. We report six additional patients with Malan syndrome and de novo NFIX deletions or sequence variants and review the 20 patients now reported. The phenotype is characterised by moderate postnatal overgrowth and macrocephaly. Median height and head circumference in childhood are 2.0 and 2.3 standard deviations (SD) above the mean, respectively. There is overlap of the facial phenotype with NSD1-positive Sotos syndrome in some cases including a prominent forehead, high anterior hairline, downslanting palpebral fissures and prominent chin. Neonatal feeding difficulties and/or hypotonia have been reported in $30 \%$ of patients. Developmental delay/learning disability have been reported in all cases and are typically moderate. Ocular phenotypes are common, including strabismus $(65 \%)$, nystagmus $(25 \%)$ and optic disc pallor/hypoplasia (25\%). Other recurrent features include pectus excavatum $(40 \%)$ and scoliosis $(25 \%)$. Eight reported patients have a deletion also encompassing CACNA1A, haploinsufficiency of which causes episodic ataxia type 2 or familial hemiplegic migraine. One previous case had episodic ataxia and one case we report has had cyclical vomiting responsive to pizotifen. In individuals with this contiguous gene deletion syndrome, awareness of possible later neurological manifestations is important, although their penetrance is not yet clear.

European Journal of Human Genetics (2015) 23, 610-615; doi:10.1038/ejhg.2014.162; published online 13 August 2014

\section{INTRODUCTION}

Overgrowth syndromes comprise a group of genetic disorders that combine height greater than or equal to two SDs above the mean with variable other features. Amongst the best recognised are Sotos syndrome (MIM 117550), Weaver syndrome (MIM 277590), Beckwith-Wiedemann syndrome (MIM 130650) and SimpsonGolabi-Behmel syndrome (MIM 312870). ${ }^{1}$ Other characteristics often present include macrocephaly, developmental delay, advanced bone age, facial dysmorphic features, hemihypertrophy, vascular malformations and, in some disorders, an increased risk of neoplasia.

Sotos syndrome is caused by haploinsufficiency of the NSD1 (Nuclear receptor Set Domain containing protein 1; MIM 606681) gene and is characterised by learning difficulties, variable medical problems including cardiac anomalies, renal anomalies and seizures and a recognisable facial gestalt, which includes dolichocephaly with a broad forehead, sparse frontotemporal hair, a prominent chin and downslanting palpebral fissures. Up to $95 \%$ of individuals with classical features of Sotos syndrome have an identifiable pathogenic
NSD1 variant, but there are a substantial number of NSD1-negative individuals with overgrowth and some Sotos-like features but who do not have the classical facial gestalt of Sotos syndrome. ${ }^{2,3}$

Recently, pathogenic de novo monoallelic variants in the NFIX (Nuclear Factor I/X; MIM 164005) gene have been identified in individuals with a Sotos-like phenotype as well as in individuals with Marshall-Smith syndrome, a disorder associated with advanced skeletal maturation, life-threatening upper airway compromise and failure to thrive. These two distinct phenotypes seem to result from two distinct classes of causative variant. Marshall-Smith syndrome has only been seen in those with frameshift and splice site variants in exons 6-8, which seem to evade nonsense-mediated RNA decay. A variety of variant types have been seen in association with the Sotos-like phenotype, including whole gene deletions (10 patients), one nonsense variant and missense variants and in-frame deletions affecting the DNA-binding domain (3 patients). ${ }^{4-10}$ Five of the reported NFIX deletions also encompass the nearby CACNA1A gene, haploinsufficiency of which causes episodic ataxia type 2 or familial hemiplegic migraine.

${ }^{1}$ Department of Paediatrics, Maastricht University Medical Center, Maastricht, The Netherlands; ${ }^{2}$ Department of Clinical Genetics, Maastricht University Medical Center Maastricht, The Netherlands; ${ }^{3}$ North East Thames Regional Genetics Service, Great Ormond Street Hospital for Children NHS Trust, London, UK; ${ }^{4}$ MRC Centre for Neuromuscular Diseases, National Hospital for Neurology and Neurosurgery, London, UK; ${ }^{5}$ Department of Paediatrics, Maxima Medical Centre, Belhaven, The Netherlands; ${ }^{6}$ Department of Clinical Genetics, Leiden University Medical Centre, Leiden, The Netherlands; ${ }^{7}$ Clinical and Molecular Genetics Unit, UCL Institute of Child Health, London, UK ${ }^{*}$ Correspondence: Dr RH Scott, Department of Clinical Genetics, Great Ormond Street Hospital for Children NHS Trust, York House, Great Ormond Street, London WC1N 3JH, UK. Tel: +44 207762 6845; Fax: +44 207813 8141; E-mail: richard.scott@gosh.nhs.uk

Received 3 March 2014; revised 11 June 2014; accepted 18 June 2014; published online 13 August 2014 
In this paper, we describe six additional patients with a Sotos-like phenotype and de novo NFIX deletions or sequence variants and review the literature.

\section{MATERIALS AND METHODS}

High-resolution aCGH was performed on genomic DNA and analysed versus reference DNA (Kreatech, Amsterdam, The Netherlands). NimbleGen $135 \mathrm{k}$ CGH microarray was used with a calculated functional resolution of $0.2 \mathrm{Mb}$ (95\% confidence limits). The DNA samples were labelled (test with Cy3 and reference with Cy5) and co-hybridised to the microarray in accordance with the manufacturer's instructions (NimbleGen Arrays User's Guide: CGH and CGH/LOH Arrays v9.1, Roche NimbleGen, Madison, WI, USA). The microarray was washed and then scanned on an Axon GenePix 4400A Scanner using GenePix Pro 7 software (Molecular Devices, Sunnyvale, CA, USA). Raw data were normalised, LOESS correction applied and the data ratios calculated using DEVA v1.01 Software (Roche NimbleGen). The normalised data were processed using Infoquant Fusion v6.0 software (Infoquant, London, UK) with analysis call settings of three consecutive probes $+/-0.4 \mathrm{Cy} 3 / \mathrm{Cy} 5$ ratio.

One patient (Patient 4) initially underwent aCGH as part of the DDD study using a custom Agilent $2 \times 1 \mathrm{M}$ array-CGH (Agilent UK, Wokingham, Berkshire, UK), with five probes per exon and a mean backbone spacing of $\sim 4 \mathrm{~kb}$. Patient samples were processed in batches of 95, labelled in Cy5 using Agilent labelling reagents (Agilent UK), and hybridised against a pool of 500 developmentally normal males labelled in Cy3. All laboratory procedures were supported by a bespoke LIMS, which interacted with DECIPHER to facilitate sample tracking. Following feature extraction, CNVs were called using a novel in-house analysis (CNsolidate) that combines 12 weighted change point detection algorithms. Patient data were annotated with allele frequencies based on overlap with a set of controls analysed on the same platform (570 population controls from the UK Blood Service and 455 developmentally normal individuals from the Scottish Family Health Study).

In all cases, for confirmation of the deletion and for parental testing, realtime quantitative PCR was carried out using primers (Sigma-Aldrich, St Louis, MO, USA) from within intron 2 of the NFIX gene. Primers were checked for specificity by melt curve analysis and run on a StepOne Plus Real Time PCR system (Life Technologies Applied Biosystems, Paisley, UK) using the SYBR Green comparative $\triangle \triangle \mathrm{CT}$ method. Primers from within genes ACTBL2 and $M A N E A$ were used as endogenous control sequence targets. Results were processed using StepOne Plus Software v2.2 with copy number loss or gain indicated by relative quantitation (RQ) values.

Conventional Sanger sequencing of the NFIX gene was performed on genomic DNA extracted from peripheral blood cells. All coding exons and intron/exon boundaries were included, based on transcript NM_002501.2. Exon numbering is as used by Malan et al. ${ }^{7}$ also based on this transcript. Primer sequences and PCR conditions are available on request. PCR products were amplified using standard protocols. Sequencing reactions were performed using the BigDye Terminator v3.1 Cycle Sequencing Kit (Applied Biosystems, Cheshire, UK) and separated on the ABI 3730 (Applied Biosystems). Results were analyzed using SeqScape v2.5 software (Applied Biosystems).

Details of the deletions identified have been submitted to the Decipher database. Details of the sequence variants have been submitted to the HGMD database.

\section{RESULTS}

\section{Case descriptions}

Patient 1. This 3-year-old boy was referred to the Clinical Genetics clinic with developmental delay and dysmorphic features. He is the first child of healthy unrelated British parents. Vaginal bleeding at 9 weeks and premature contractions at 28 weeks gestation complicated the pregnancy. His birth weight was $2490 \mathrm{~g}$ at 37 weeks gestation $(-1.3$ SD below the mean; UK90 growth charts). Motor milestones were delayed. He sat unsupported at 1 year, crawled at 18 months and walked at around 2.5-3 years. At the age of 3, he spoke several words, no sentences. He had a prominent forehead and chin and slightly downslanting palpebral fissures (Figure 1). He had plagiocephaly and mild facial asymmetry. Head circumference was $52 \mathrm{~cm}(+0.3 \mathrm{SD})$, height $103 \mathrm{~cm}(+1.9 \mathrm{SD})$ and weight $19.3 \mathrm{~kg}(+2.6 \mathrm{SD})$. There was mild scoliosis of the thoracic spine with $1 \mathrm{~cm}$ leg asymmetry and a mild pectus excavatum. Bone age was 3.5 years at a chronological age of 3 years (1 SD above mean). On the basis of these clinical features, he was given a working diagnosis of Sotos syndrome. NSD1 sequencing and karyotype were normal. At last follow-up, aged 10 years, his height was $152.0 \mathrm{~cm}(+2.2 \mathrm{SD})$, his weight $45.5 \mathrm{~kg}(+1.7$ SD) and his head circumference $55.5 \mathrm{~cm}(+0.7$ SD). He had behavioural difficulties and a diagnosis of autistic spectrum disorder.

Patient 2. This 3.5-year-old girl was referred to the Clinical Genetics clinic with global developmental delay, hypotonia and overgrowth. She is the only child of healthy unrelated Chinese parents and was born by forceps delivery at 42 weeks gestation. She first sat unsupported at the age of 12 months and made her first independent steps by the age of 2.5 years. Aged 3.5 years, she said 'dada', but no other clear words. On physical examination, at age 2.5 years, her height was $94.5 \mathrm{~cm}(+1.0 \mathrm{SD}$ using British charts), head circumference $51.1 \mathrm{~cm}(+1.0 \mathrm{SD})$ and weight $14.1 \mathrm{~kg}(+0.7 \mathrm{SD})$. She had scaphocephaly, a prominent forehead, depressed nasal bridge and deep-set eyes. Her palate was high arched and there was significant drooling. There was no scoliosis.

Patient 3. This 2-years and 8-month-old girl was referred to the Clinical Genetics clinic because of abnormal array CGH results. Array was performed because of global developmental delay and oculomotor dyspraxia. She had had one febrile seizure. She was born at term after an uneventful pregnancy to healthy unrelated Italian parents weighing $3.6 \mathrm{~kg}(+0.5 \mathrm{SD})$. She first walked at 2.5 years. She had been found to have virtually undetectable biotinidase levels for which biotin was given for a brief period. A repeat measurement of biotinidase levels - several months after biotin treatment was stopped - was normal. Her height was $93.4 \mathrm{~cm}(+0.5 \mathrm{SD})$, head circumference $52 \mathrm{~cm}(+1.6 \mathrm{SD})$ and weight $15.4 \mathrm{~kg}(+1.3 \mathrm{SD})$. She has intermittent end-gaze horizontal nystagmus and an intermittent convergent squint. She had a prominent forehead and slightly downslanted palpebral fissures. A brain MRI showed no abnormalities.

Patient 4. This 13-month-old boy was referred to the Clinical Genetics clinic because of global developmental delay and overgrowth. $\mathrm{He}$ is the only child of healthy parents of British and Afro-Caribbean ancestry. He was born at 39 weeks gestation weighing $4.1 \mathrm{~kg}(+1.1$ $\mathrm{SD})$. At birth, he was hypertonic with flexed posture and adducted thumbs. There were mild contractures of small joints, which resolved spontaneously in the first year of life. He had global developmental delay, first standing up at 2.5 years, walking at 4.5 years. At the age of 5.5 years, he could babble but had no words. There was a left divergent squint and a visual evoked potential examination showed post-retinal dysfunction bilaterally. At the age of 4 years, he presented with cyclical vomiting. This resolved following commencement of pizotifen. He had scaphocephaly and a prominent forehead. Aged 5.5 years, his height was $128 \mathrm{~cm}(+2.8 \mathrm{SD})$, his head circumference $59 \mathrm{~cm}(+2.9 \mathrm{SD})$ and his weight $30.5 \mathrm{~kg}(+3.9 \mathrm{SD})$. Several investigations had been performed, including plasma creatinine kinase, very long chain fatty acids, karyotype, $1 \mathrm{Mb}$ resolution array CGH, and PTEN and NSD1 sequencing. All results were normal. A brain MRI performed at the age of 8 weeks showed delayed myelination. 

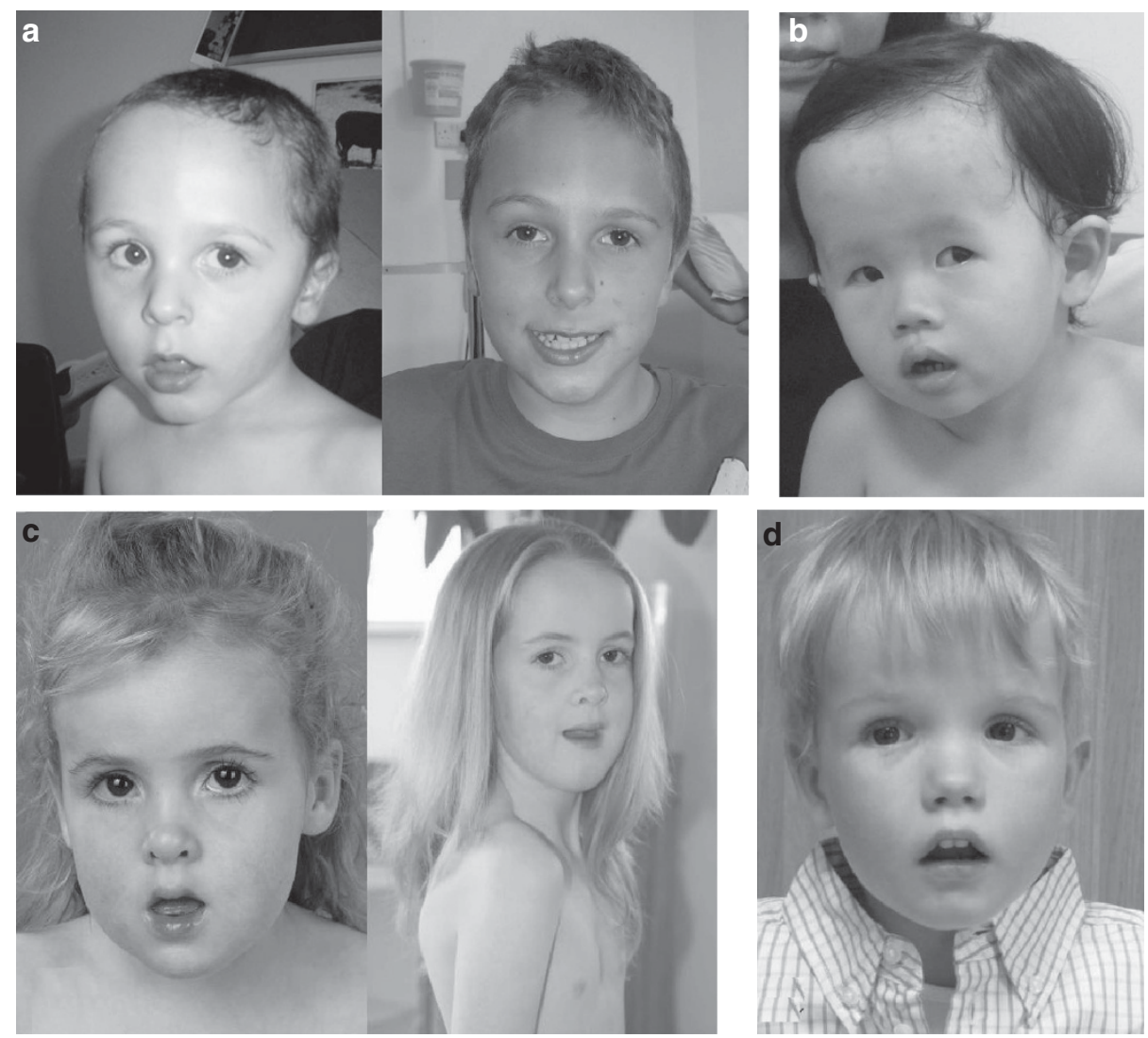

Figure 1 Facial features of patients. (a) Patient 1 at age 3 and 10; (b) Patient 2 at age 3.5; (c): Patient 5 at age 2.5 and 7. Note the pectus excavatum at 7 years; (d) Patient 6 at age 3.

Patient 5. This 2-years and 5-month-old girl was referred to the Clinical Genetics clinic because of global developmental delay and hypotonia. She was born at term to healthy unrelated Dutch parents. Her birth weight was reported as 'average'. She had an uneventful neonatal period, except for muscular hypotonia. There was a global developmental delay with walking and several single words at 2 years. There were recurrent upper airway infections for which grommets were inserted. Physical examination showed macrocephaly with a head circumference of $52 \mathrm{~cm}(+3.3 \mathrm{SD})$ and height and weight were both 2-3 SD above the mean. There was frontal bossing, mild downslanting palpebral fissures, a small mouth, pointed chin and a pectus excavatum. Several investigations had been performed, including karyotype, MLPA of subtelomeric regions and microdeletions, high-resolution array and NSD1 sequencing, all of which gave normal results. Bone age was advanced. A brain MRI performed at the age of 3 years showed periventricular white matter hyperintensity, slightly enlarged ventricles, possible heterotopias and a persistent cavum septum pellucidum. Sanger sequencing of NFIX was requested based on this clinical presentation.

Patient 6. This 3-year-old boy was referred to the Clinical Genetics clinic because of macrocephaly and developmental delay. He was born at term to healthy unrelated Dutch parents after an uneventful pregnancy. His birth weight was $3.25 \mathrm{~kg}$ at term $(-0.3 \mathrm{SD})$. During the first few months of his life, he suffered from gastro-oesophageal reflux disease, which resolved with medication. His development was delayed with walking at 2.5-3 years and first single words at the age of 2.5. On physical examination, he had macrocephaly with a mild facial asymmetry and downslanting palpebral fissures. Head circumference was +1.0 SD, height was +0.7 SD and weight was on the 50th percentile. Previously, several investigations were performed, including a high-density array and FMR1 expansion testing and NSD1 sequencing. A brain MRI performed at the age of 2 showed no abnormalities. The bone age was 3.5-4 years at the age of 3. Sanger sequencing of NFIX was requested based on this clinical presentation.

\section{Molecular results}

Array CGH detected deletions in Patients 1-4 (Table 1). In all patients, the deletion had arisen de novo, as confirmed by parental array CGH, FISH and/or quantitative PCR. Array CGH in Patient 1 revealed a 110-170-kb deletion on chromosome 19: chr19.hg19:g. $\left(13,060,729 \_13,097,782\right) \_\left(13,209,504 \_13,232,425\right)$. The deletion minimally encompasses the whole NFIX gene. Maximally, it also deletes or partially deletes six other RefSeq genes with no known haploinsufficiency phenotype. Array CGH on Patient 2 showed a larger deletion of approximately 740-810 kb chr19.hg19:g.(12,724,476 _12,774,135)_(13,511,059_13,534,643). This deletion encompasses the whole NFIX gene and all but the final three exons of CACNA1A as well as between 32 and 34 other RefSeq genes with no known haploinsufficiency phenotype. FISH analysis using probe RP11-35L15 confirmed the presence of this deletion. Array CGH on Patient 3 showed a 410-470-kb deletion chr19.hg19:g.(13,060,729_13,097,782)_ $\left(13,511,059 \_13,534,643\right)$. This deletion encompasses the whole NFIX gene and all but the final three exons of CACNA1A as well as between five and seven other RefSeq genes with no known haploinsufficient phenotype. Array CGH on Patient 4 identified a 560-640-kb deletion chr19.hg19:g.(12,745,959_12,812,594)_(13,376,997_13,383,138). This deletion encompasses NFIX and the first 26 exons of CACNA1A 
Table 1 Clinical features and details of pathogenic NFIX deletions and sequence variants in Patients 1-6

\begin{tabular}{|c|c|c|c|c|c|c|}
\hline & Patient 1 & Patient 2 & Patient 3 & Patient 4 & Patient 5 & Patient 6 \\
\hline $\begin{array}{l}\text { Mechanism } \\
\text { Genes deleted/affected }\end{array}$ & $\begin{array}{l}\text { Deletion } \\
\text { NFIX }\end{array}$ & $\begin{array}{c}\text { Deletion } \\
N F I X+C A C N A 1 A^{a}\end{array}$ & $\begin{array}{c}\text { Deletion } \\
N F I X+C A C N A 1 A^{a}\end{array}$ & $\begin{array}{c}\text { Deletion } \\
N F I X+C A C N A 1 A^{a}\end{array}$ & $\begin{array}{l}\text { Frameshift variant } \\
\text { NFIX }\end{array}$ & $\begin{array}{c}\text { Nonsense variant } \\
\text { NFIX }\end{array}$ \\
\hline Minimal breakpoints/variant & $\begin{array}{c}13097 \\
782 \_13209504\end{array}$ & $12724476 \_13511059$ & 13097 782_13511059 & 12812594_13376997 & $\begin{array}{l}\text { c.90_99del, } \\
\text { p.(Trp30fs) }\end{array}$ & $\begin{array}{l}\text { c. } 1012 C>T, \\
\text { p. }\left(G \ln 338^{a}\right)\end{array}$ \\
\hline Size & $110-170 \mathrm{~kb}$ & $740-810 \mathrm{~kb}$ & $410-470 \mathrm{~kb}$ & $560-640 \mathrm{~kb}$ & - & - \\
\hline Age at last assessment & 10.0 years & 3.5 years & 2.7 years & 5.5 years & 5 years & 3 years \\
\hline Birthweight (SD) & -1.3 & N/A & +0.5 & +1.1 & N/A & -0.25 \\
\hline Length/height (SD) ${ }^{b}$ & +2.2 & +1.0 & +0.5 & +2.8 & $+2-3$ & +0.7 \\
\hline Weight $(S D)^{\mathrm{b}}$ & +1.7 & +1.0 & +1.3 & +2.9 & +2.3 & 0 \\
\hline $\mathrm{OFC}(\mathrm{SD})^{\mathrm{b}}$ & +0.7 & +0.7 & +1.6 & +3.9 & +3.3 & +1.0 \\
\hline Dysmorphic features & $\begin{array}{l}\text { Plagiocephaly, } \\
\text { prominent fore- } \\
\text { head, facial } \\
\text { asymmetry, low } \\
\text { posterior hairline }\end{array}$ & $\begin{array}{l}\text { Scaphocephaly, promi- } \\
\text { nent forehead, depressed } \\
\text { nasal bridge, deep-set } \\
\text { eyes, high arched palate }\end{array}$ & $\begin{array}{l}\text { Prominent forehead, } \\
\text { slightly downslanting } \\
\text { palpebral fissures }\end{array}$ & $\begin{array}{l}\text { Scaphocephaly, promi- } \\
\text { nent forehead }\end{array}$ & $\begin{array}{l}\text { Prominent forehead, } \\
\text { slightly downslanting } \\
\text { palpebral fissures, } \\
\text { elongated face, small } \\
\text { mouth, pointed chin }\end{array}$ & $\begin{array}{l}\text { Frontal bossing, } \\
\text { mild facial } \\
\text { asymmetry, } \\
\text { slightly down- } \\
\text { slanting palpeb- } \\
\text { ral fissures }\end{array}$ \\
\hline Developmental delay & Moderate & Moderate & Moderate & Severe & Severe & Severe \\
\hline $\begin{array}{l}\text { Neonatal feeding difficul- } \\
\text { ties/hypotonia }\end{array}$ & - & - & - & - & - & - \\
\hline Seizures & + & - & - & - & - & - \\
\hline Episodic ataxia & - & - & - & - & - & - \\
\hline Migraine & - & - & - & + & - & - \\
\hline Nystagmus & - & + & - & - & - & - \\
\hline Strabismus & - & + & + & + & + & + \\
\hline Optic disc pallor/hypoplasia & - & - & - & - & - & - \\
\hline Advanced bone age & - & $\mathrm{N} / \mathrm{A}$ & $\mathrm{N} / \mathrm{A}$ & $\mathrm{N} / \mathrm{A}$ & + & $\mathrm{N} / \mathrm{A}$ \\
\hline Scoliosis & + & - & - & - & - & - \\
\hline Pectus excavatum & + & + & + & - & + & - \\
\hline
\end{tabular}

Abbreviations: OFC, occipital-frontal circumference; N/A, not available; SD, standard deviations above (+) or below ( $(-)$ mean.

a Also deleted in all cases are five annotated genes with no known human phenotype located between NFIX and CANCA1A (LYL1, TRMT1, NACC1, STX10 and IER2). Genomic positions are given according to build HG19.

beasurement at last assessment in clinic.

and between 26 and 31 other Refseq genes with no known haploinsufficiency phenotype.

In Patient 5, a de novo heterozygous frameshift variant was identified in the NFIX gene: c.90_99del, p.(Trp30fs). In Patient 6, a de novo heterozygous nonsense variant was identified in exon 7 of the NFIX gene: c. $1012 \mathrm{C}>\mathrm{T}$, p. $\left(\mathrm{G} \ln 338^{\star}\right)$.

\section{DISCUSSION}

Recently, there have been several reports of patients with Sotos-like features who carry a de novo sequence variant or deletion of NFIX at 19p13.2.5,7-11 Here, we report six additional patients with this disorder who have de novo disruption of the NFIX gene by either deletions (Patients 1-4; Table 1) or sequence variants (Patients 5 and 6). In three of the four deletion patients, there is also deletion of CACNA1A. This brings the total number of patients reported with the Sotos-like overgrowth disorder caused by NFIX to 20, including 14 with deletions (8 encompassing CACNA1A), 3 with truncating variants and 3 with missense substitutions or in-frame deletions (Table 2 and Supplementary Table 1). With 20 patients now reported and a distinctive phenotype emerging, we believe the condition is best referred to as Malan syndrome. ${ }^{7}$

The phenotype is characterised by mild-to-moderate overgrowth, macrocephaly with a prominent forehead and moderate developmental delay, all features of NSD1-positive Sotos syndrome (Figure 1, Tables 1 and 2 and Supplementary Table 1). Birth weight is often within normal limits among Malan syndrome patients with the median birth weight $0.3 \mathrm{SD}$ above the mean (range -2.3 to +2.1 $\mathrm{SD})$. Overgrowth is therefore often postnatal in onset and is typically less marked than in Sotos syndrome with a median height in Malan syndrome of $2.0 \mathrm{SD}$ above the mean (range -0.5 to $+3.8 \mathrm{SD}$ ) compared to a median of $>+2.5 \mathrm{SD}$ in Sotos syndrome (Table 3). ${ }^{12-14}$ Median head circumference is $2.3 \mathrm{SD}$ above the mean (range -0.7 to $3.9 \mathrm{SD}$ ) and may also be more mildly increased than in Sotos syndrome where it is $>+2.5 \mathrm{SD}$. Median weight is $0.6 \mathrm{SD}$ above the mean (range -0.9 to $+2.9 \mathrm{SD}$ ). Developmental delay has been reported in all cases to date and was reported as moderate in 15 $(75 \%)$, severe in $4(20 \%)$ and mild in $1(5 \%)$. Autistic traits have been reported in five cases (25\%). A common early presentation is with neonatal hypotonia and/or feeding difficulties, which have been specifically commented upon in five patients (25\%). Seizures have been reported in five patients (25\%).

A number of the reported cases have facial features overlapping those seen in Sotos syndrome including prominent chin and downslanting palpebral fissures and prominent forehead with a high anterior hairline (Figure 1). However, of our cases, only Patient 1 had sufficient features to have led to a working diagnosis of Sotos syndrome prior to molecular testing. As in Sotos syndrome, advanced bone age is common, present in six of seven from whom data are available. ${ }^{13}$ Scoliosis is also a feature of both conditions, reported in five Sotos-like NFIX patients (25\%). ${ }^{12,14}$

There are often additional findings not commonly seen in classical Sotos syndrome including strabismus (13 patients; 65\%; Table 3), 
Table 2 Clinical features of Malan syndrome patients with pathogenic NFIX variants and deletions reported here and in the literature

\begin{tabular}{|c|c|c|c|c|c|}
\hline & All & $\begin{array}{c}\text { Deletion of NFIX and } \\
\text { other genes including CACNA1A }\end{array}$ & $\begin{array}{c}\text { Deletion of NFIX } \\
\text { excluding CACNA1A }\end{array}$ & $\begin{array}{c}\text { Nonsense or } \\
\text { frameshift NFIX variant }\end{array}$ & $\begin{array}{c}\text { Missense or in-frame } \\
\text { NFIX variant }\end{array}$ \\
\hline Number of patients & 20 & 8 & 6 & 3 & 3 \\
\hline Median birthweight (SD) & +0.3 & 0 & +0.9 & +0.2 & -0.4 \\
\hline Median length/height (SD) ${ }^{a}$ & +2.0 & +1.4 & +1.7 & +2.5 & +3.8 \\
\hline Median weight (SD) & +0.6 & +1.2 & +1.2 & +1.3 & +0.6 \\
\hline Median OFC (SD) & +2.3 & +2.1 & +2.3 & +2.5 & +2.8 \\
\hline \multicolumn{6}{|l|}{ Developmental delay } \\
\hline Mild & $1(5 \%)$ & 0 & 0 & 0 & 1 \\
\hline Moderate & $15(75 \%)$ & 7 & 5 & 2 & 1 \\
\hline Severe & $4(20 \%)$ & 1 & 1 & 1 & 1 \\
\hline Neonatal feeding difficulties/hypotonia & $6 / 20(30 \%)$ & $2 / 8$ & $3 / 6$ & $0 / 3$ & $1 / 3$ \\
\hline Seizures & $5 / 20(25 \%)$ & $1 / 8$ & $3 / 6$ & $0 / 3$ & $1 / 3$ \\
\hline Episodic ataxia & $1 / 20(5 \%)$ & $1 / 8$ & $0 / 6$ & $0 / 3$ & $0 / 3$ \\
\hline Migraine & $1 / 20(5 \%)$ & $1 / 8$ & $0 / 6$ & $0 / 3$ & $0 / 3$ \\
\hline Nystagmus & $5 / 20(25 \%)$ & $2 / 8$ & $1 / 6$ & $1 / 3$ & $1 / 3$ \\
\hline Strabismus & $13 / 20(65 \%)$ & $5 / 8$ & $4 / 6$ & $2 / 3$ & $2 / 3$ \\
\hline Optic disc pallor/hypoplasia & $5 / 20(25 \%)$ & $1 / 8$ & $3 / 6$ & $0 / 3$ & $1 / 3$ \\
\hline Advanced bone age & $6 / 7(86 \%)$ & $1 / 1$ & $2 / 3$ & $2 / 2$ & $2 / 2$ \\
\hline Scoliosis & $5 / 20(25 \%)$ & $0 / 8$ & $2 / 6$ & $1 / 3$ & $2 / 3$ \\
\hline Pectus excavatum & $8 / 20(40 \%)$ & $2 / 8$ & $3 / 6$ & $1 / 3$ & $2 / 3$ \\
\hline
\end{tabular}

Abbreviations: OFC, occipital-frontal circumference; N/A, not available; SD, standard deviations above (+) or below ( - ) mean. Genomic positions are given according to the HG19 build.

a Measurement at last assessment in clinic.

nystagmus (5 patients; 25\%) and optic disc pallor/nerve hypoplasia (5 patients; 25\%). ${ }^{12,13}$ Pectus excavatum, uncommon in Sotos syndrome, was present in eight patients (40\%) and may be a particularly useful discriminating feature (Table 3 ). We note that mice deficient in Nfix function show, in addition to brain abnormalities, a kyphotic deformation of the spine and impairment of endochondral ossification in the vertebrae and femur. ${ }^{15,16}$

There have been no reports of neoplasia in the condition to date. The small number of cases reported to date hinders identification of genotype-phenotype correlations for NFIX. However, a strong correlation has been observed between the classes of variant that cause Malan syndrome and Marshall-Smith syndrome, with Marshall-Smith syndrome seen only with frameshift or splice site variants in exons 6-8. It has been speculated that these evade nonsense-mediated RNA decay and it is this that results in their distinct phenotypic effect. It should also be noted that there are several alternative transcripts of the NFIX gene, which may impact on the differential phenotypic effects of variants. Amongst our patients is one child with a Sotos-like phenotype (Patient 6, Figure 1) and a nonsense variant in exon 7 (c.1012C $\left.>\mathrm{T}, \mathrm{p} .\left(\mathrm{G} \ln 338^{\star}\right)\right)$. The same nucleotide is involved in frameshift variants identified in MarshallSmith syndrome patients (c.1011_1012delTC and c.1008_ 1012delCTCTC). ${ }^{7}$ Suitable samples were not available from our patient to determine if the mutated transcript evades nonsensemediated RNA decay. Nevertheless, our finding urges caution regarding division of phenotypic effect based on variant position alone.

In the large majority of cases, there appears to be clear separation of the phenotype of patients, with patients either manifesting with the Malan syndrome or Marshall-Smith syndrome. However, we note that the patient with a 21-nucleotide deletion in the DNA-binding domain reported by Priolo et $a l^{8}$ had a combination of a Sotos-like presentation and mild features of Marshall-Smith syndrome
Table 3 Features that assist in distinguishing Malan syndrome patients from those with Sotos syndrome. ${ }^{12,13}$

\begin{tabular}{lcc}
\hline & Malan syndrome & $\begin{array}{c}\text { Sotos } \\
\text { syndrome }\end{array}$ \\
\hline Median height (SD) & +2.0 & $>+2.5$ \\
Median head circumference (SD) & +2.3 & $>+2.5$ \\
Pectus excavatum & $40 \%$ & $<15 \%$ \\
Strabismus & $65 \%$ & $<15 \%$ \\
Ocular nerve hypoplasia/pallor & $25 \%$ & $<15 \%$ \\
Cerebellar ataxia, hemiplegic migraine & At risk if CACNA1A deleted & -
\end{tabular}

Abbreviation: SD, standard deviations above the mean

including sleep apnoea and bullet-shaped phalanges, suggesting that this separation may not be absolute.

In all of the cases we report, the NFIX variants occurred de novo. This is the case in the large majority of cases tested and it is clear that the penetrance of the disorder is high. In one family, there was presumed gonadal mosaicism. ${ }^{9}$ Yoneda et al $l^{6}$ reported one child with a missense substitution in the DNA-binding domain which had been inherited from a possibly affected mother (c.362G $>$ C, p.(Arg121Pro)); Supplementary Table 2). Although the location and physicochemical properties of this variant and the description of the facial and other phenotypic features of this child are consistent with its pathogenicity, caution is required in the interpretation of missense variants in NFIX, particularly when they are inherited.

The literature describes deletions or truncating variants of CACNA1A as typically causing episodic ataxia type 2 (EA2, MIM108500). ${ }^{17}$ Pathogenic missense variants typically cause familial hemiplegic migraine type 1 (FHM1, MIM141500), in which cerebellar signs and symptoms varying from isolated nystagmus to progressive 
ataxia may also be found. ${ }^{18}$ Overlap exists between the two phenotypes and hemiplegic migraine has been reported in individuals with truncating variants or deletions. ${ }^{19,20}$ CAGexpansion in exon 47 of CACNA1A causes the distinct disorder spinocerebellar ataxia 6 (SCA6, MIM 183086), characterised by adultonset, slowly progressive cerebellar ataxia, dysarthria and nystagmus.

Eight patients have now been reported with contiguous deletion of NFIX and CACNA1A. The patients have mainly presented with features attributable to NFIX rather than CACNA1A. One of our patients (Patient 4) aged 4 years presented with cyclical vomiting responsive to pizotifen and one of the patients reported in the literature aged 9 years had episodic ataxia. Nystagmus has been seen in a similar number of NFIX patients with and without deletions, and it is likely that in most, it is not a feature of EA2/FHM1 but a result of NFIX haploinsufficiency.

Lifetime penetrance for EA2 is thought to be $80-90 \%$ but there is a wide range of age of onset - between 2 and 32 years - and it has been suggested that deletions may have an older age of onset. ${ }^{21}$ The absence of features of EA2/FHM1 in the majority of NFIX-CACNA1A deletion cases may simply reflect the young age of those reported to date (the median age at last assessment is 9 years). Longer-term follow-up of patients with NFIX-CACNA1A will provide data on the (age-related) penetrance of CACNA1A deletion ascertained independent of family history and may differ from estimates available from familial EA2 and FHM1 cohorts. Until these data are available, caution is advisable when estimating the penetrance of deletion of CACNA1A in NFIX-CACNA1A deletion patients. ${ }^{19,21,22}$

A number of other genes are deleted in addition to NFIX and CACNA1A in the patients others and we have reported. To date no other obvious genotype-phenotype correlations have emerged. However, we do note that the child with the largest reported deletion, measuring $\sim 3 \mathrm{Mb}$, had a more complicated phenotype including craniosynostosis and was growth retarded. ${ }^{10}$

There is little overlap in breakpoints between the reported deletions and there is no clustering of breakpoints around low-copy repeats (LCRs) as in a number of common microdeletion/duplication disorders. It is likely that other mechanisms are responsible, for example, non-allelic homologous recombination between Alu elements. Alu elements comprise an estimated $10-15 \%$ of the human genome but are overrepresented on chromosome 19, comprising $25 \%$ of its sequence. ${ }^{22,23}$ They mediate a number of reported copy number aberrations on chromosome 19 and occupy $30 \%$ of the genomic footprint of $C A C N A 1 A$, supporting a possible role in NFIX-CACNA1A deletions.

In conclusion, NFIX analysis should be considered in patients presenting with overgrowth, macrocephaly and developmental delay including those in whom Sotos syndrome has been considered clinically but are negative for pathogenic NSD1 variants. Medical follow-up should include developmental support, ophthalmological assessment, surveillance for scoliosis and a low threshold for investigation of possible seizures. In cases where CACNA1A is deleted together with NFIX, awareness of possible later neurological manifestations is important.

\section{CONFLICT OF INTEREST}

The authors declare no conflict of interest.

\section{ACKNOWLEDGEMENTS}

We thank the patients and their parents. M.K. is supported by a grant from the academic fund ('Academisch Fonds') of the Maastricht University Medical Centre. The DDD study presents independent research commissioned by the Health Innovation Challenge Fund [grant number HICF-1009-003], a parallel funding partnership between the Wellcome Trust and the Department of Health, and the Wellcome Trust Sanger Institute (grant number WT098051). The views expressed in this publication are those of the author(s) and not necessarily those of the Wellcome Trust or the Department of Health. The study has UK Research Ethics Committee approval (10/H0305/83, granted by the Cambridge South REC, and GEN/284/12 granted by the Republic of Ireland REC). The research team acknowledges the support of the National Institute for Health Research, through the Comprehensive Clinical Research Network.

1 Neylon OM, Werther GA, Sabin MA: Overgrowth syndromes. Curr Opin Pediatr 2012; 24: 505-511.

2 Douglas J, Hanks S, Temple IK et al: NSD1 mutations are the major cause of Sotos syndrome and occur in some cases of Weaver syndrome but are rare in other overgrowth phenotypes. Am J Hum Genet 2003; 72: 132-143.

3 De Boer L, Van Duyvenvoorde HA, Willemstein-Van Hove EC et al: Mutations in the NSD1 gene in patients with Sotos syndrome associate with endocrine and paracrine alterations in the IGF system. Eur J Endocrinol 2004; 151: 333-341.

4 Auvin S, Holder-Espinasse M, Lamblin MD, Andrieux J: Array-CGH detection of a de novo 0.7-Mb deletion in 19p13.13 including CACNA1A associated with mental retardation and epilepsy with infantile spasms. Epilepsia 2009; 50: 2501-2503.

5 Dolan M, Mendelsohn NJ, Pierpont ME, Schimmenti LA, Berry SA, Hirsch B: A novel microdeletion/microduplication syndrome of 19p13.13. Genet Med 2010; 12: 503-511.

6 Yoneda Y, Saitsu H, Touyama M et al: Missense mutations in the DNA-binding/dimerization domain of NFIX cause Sotos-like features. J Hum Genet 2012; 57: 207-211.

7 Malan V, Rajan D, Thomas S et al: Distinct effects of allelic NFIX mutations on nonsense-mediated mRNA decay engender either a Sotos-like or a Marshall-Smith syndrome. Am J Hum Genet 2010; 87: 189-198.

8 Priolo M, Grosso E, Mammi C et al: A peculiar mutation in the DNA-binding/ dimerization domain of NFIX causes Sotos-like overgrowth syndrome: a new case. Gene 2012; 511: 103-105.

9 Nimmakayalu M, Horton VK, Darbro B et al: Apparent germline mosaicism for a novel $19 p 13.13$ deletion disrupting NFIX and CACNA1A. Am J Med Genet A 2013; 161: 1105-1109.

10 Lysy PA, Ravoet M, Wustefeld S et al: A new case of syndromic craniosynostosis with cryptic 19p13.2-p13.13 deletion. Am J Med Genet A 2009; 149A: 2564-2568.

11 Lehman AM, du Souich C, Chai D et al: 19p13.2 microduplication causes a Sotos syndrome-like phenotype and alters gene expression. Clin Genet 2010; 81: 56-63.

12 Tatton-Brown K, Cole TRP, Rahman N: Sotos syndrome; in Pagon RA, Adam MP, Ardinger HH, Bird TD, Dolan CR, Fong CT, Smith RJH, Stephens K (eds): GeneReviews [Internet]. Seattle, WA: University of Washington, 1993-2014 (17 December 2004 (updated 8 March 2012)).

13 Tatton-Brown K, Rahman N: Clinical features of NSD1-positive Sotos syndrome. Clin Dysmorphol 2004; 13: 199-204.

14 Baujat G, Cormier-Daire V: Sotos syndrome. Orphanet J Rare Dis 2007; 2: 36.

15 Bonaglia MC, Marelli S, Novara F et al: Genotype-phenotype relationship in three cases with overlapping 19p13.12 microdeletions. Eur J Hum Genet 2010; 18: 1302-1309.

16 Driller K, Pagenstecher A, Uhl M et al: Nuclear factor I X deficiency causes brain malformation and severe skeletal defects. Mol Cell Biol 2007; 27: 3855-3867.

17 Pietrobon D: Insights into migraine mechanisms and CaV2.1 calcium channel function from mouse models of familial hemiplegic migraine. J Physiol 2010; 588: 1871-1878.

18 Di Cristofori A, Fusi L, Gomitoni A, Grampa G, Bersano A: R583Q CACNA1A variant in SHM1 and ataxia: case report and literature update. J Headache Pain 2012; 13: 419-423.

19 Labrum RW, Rajakulendran S, Graves TD et al: Large scale calcium channel gene rearrangements in episodic ataxia and hemiplegic migraine: implications for diagnostic testing. J Med Genet 2009; 46: 786-791.

20 Spacey S: Episodic Ataxia Type 2; In: Pagon RA, Adam MP, Ardinger HH, Bird TD, Dolan CR, Fong CT, Smith RJH, Stephens K (eds): GeneReviews [Internet]. Seattle (WA): University of Washington, 1993-2014. (24 February 2003 (updated 8 December 2011)).

21 Riant $\mathrm{F}$, Lescoat $\mathrm{C}$, Vahedi $\mathrm{K}$ et al: Identification of CACNA1A large deletions in four patients with episodic ataxia. Neurogenetics 2009; 11: 101-106.

22 Wan J, Mamsa H, Johnston JL et al: Large genomic deletions in CACNA1A cause episodic ataxia type 2. Front Neurol 2011; 2: 51

23 Cordaux R, Hedges DJ, Batzer MA: Retrotransposition of Alu elements: how many sources? Trends Genet 2004; 20: 464-467.

Supplementary Information accompanies this paper on European Journal of Human Genetics website (http://www.nature.com/ejhg) 